\title{
Pouvoirs locaux et organisation du territoire des cités phéniciennes sous l'Empire perse achéménide
}

\author{
JOSETTE ELAYI *
}

\begin{abstract}
RESUMEN
ABSTRACT

Este artículo trata de las ciudades

This paper deals with the Phoenician

fenicias y de la organización de sus local powers and the organization of their territories during the Iron Age III/Persian Period (vith-Ivth B.C.). A geographical description of the Phoenician city-states is proposed from the present state of documentation. The socio-political structures of these cities are studied and characterized in comparison with the neighbouring Near Eastern states and the contemporaneous Greek cities (poleis). The place of the Phoenician cities in the political framework of the Persian empire is also analysed, in particular the relations between the central power and the local autonomous powers.
\end{abstract}

Les Phéniciens sont, dans l'actuelle décennie, au centre d'une nouvelle littérature écrite par des spécialistes pour un large public, qui hésite entre deux visions extrêmes: la première totalement négative (les Phéniciens

* CNRS París. 
ont-ils existé?) et la seconde qui leur donne une dimension quasi-planétaire (l'»Univers phénicien", les "Phéniciens, marins des cinq continents") ' . La question de la définition de la Phénicie et des Phéniciens est souvent posée, mais elle n'aboutit pas à des réponses claires et cohérentes. On confond notamment des notions hétérogènes telles que les entités politiques phéniciennes et puniques (cités-Etats du Proche-Orient, établissements de différents types fondés par ces cités, établissements secondaires fondés par ces établissements), la présence permanente ou temporaire de Phéniciens (groupes ou individus) dans des entités politiques autres, la géographie linguistique qui ne coïncide pas nécessairement avec la géographie politique, et la diffusion directe (ou indirecte) de la culture matérielle phénicienne qui n'est pas toujours identifiée comme telle.

En fonction des données existantes, nous avons proposé un ensemble de critères opérationnels (toujours susceptibles d'être complétés) pour identifier des «sites phéniciens», c'est-à-dire des sites sous domination politique phénicienne, dans une région et à une époque données: dans cet article, la zone littorale du Proche-Orient à l'époque de la domination perse achéménide ou Fer III ( $\mathrm{VI}^{\mathrm{e}}-\mathrm{IV}$ siècles avant J.-C.) ${ }^{2}$. C'est dans ce cadre espace-temps clairement délimité que la forme de ces pouvoirs locaux et l'organisation de leurs territoires peuvent être étudiés ${ }^{3}$.

La géographie de la Phénicie n'est pas facile à définir car les Phéniciens ne formaient pas une nation au sens où nous l'entendons aujourd'hui: la Phénicie était en effet constituée par un ensemble de cités-Etats aux territoires morcelés. A l'époque du Fer III/Perse, les changements territoriaux paraissent avoir été relativement peu importants. Ces cités étaient installées sur la façade méditerranéenne du socle syro-arabe connu sous le nom de "Croissant fertile" ${ }^{4}$. Les principales cités formaient un noyau central que nous avons appelé par commodité Phénicie centrale: Sidon, Sour/Tyr, Arwad/Arados, Gubal/Byblos, Atar/Tripolis et peut-être Beruta/Berytos (voir carte). Les plus puissantes d'entre elles (Sidon, Tyr et Arwad) entreprirent à cette époque une expansion territoriale dans les régions situées au nord et au sud de la Phénicie centrale; elles dominaient

Voir mes recensions dans Trans 5, 1992, págs. 166-171; 8, 1994, págs. 169-171; 11, 1996, págs. 147-151, 165-170 (avec bibliographie).

J. ELAYI, «Studies in Phoenician Geography during the Persian Period", JNES 41, 1982, págs. 83-110; id., Economie des cités phéniciennes sous l'Empire perse, Napoli 1990, págs. 10-30.

3 Sur la méthodologie, cf. J. ELAYI et J. SAPIN, Nouveaux regards sur la Transeuphratène, Paris 1991, págs. 173-186.

${ }^{4}$ E. DE VAumas, Le Liban, Paris 1954; P. SANLAVILLE, Etude géomorphologique de la région littorale du Liban, Beyrouth 1977. 
en Phénicie du sud Tell Megadim/Qarta, Dor, La Tour-de-Straton, Jaffa/Joppè et Ascalon, et peut-être aussi Tell Abou Hawam, Tel Shiqmona/Sycaminon, Tell Mevorach et Tel Michal/Makmish, et en Phénicie du nord au moins cinq sites: Soukas, Gabala, Sigôn, Al Mina et Myriandros. Cette définition géographique provisoire des cités phéniciennes à l'époque perse semble rejoindre à peu près celle d'Hérodote, qui avait somme toute une notion assez exacte de leur étendue territoriale. Elles présentaient un certain nombre de caractéristiques territoriales communes: c'était des cités côtières bâties en partie sur des îles (Arwad, Tripolis, Tyr, Sidon) ou sur des promontoires rocheux (Byblos, Berytos). Le premier avantage de ce type de site était de faciliter la défense: ainsi, la position insulaire de Tyr lui a permis de résister aux sièges des conquérants asiatiques successifs jusqu'à ce qu'Alexandre ait eu l'idée de relier l'île au continent. Son deuxième avantage était d'offrir aux ports une meilleure protection contre les vents dominants: les cités avaient au moins deux ports, orientés différemment pour assurer une protection contre les différents vents; les cordons de récifs servaient à installer des môles et brise-lames sans nécessité de construire en eau profonde ${ }^{5}$. Elles étaient souvent bâties à proximité de collines rocheuses qui servaient de carrières ou de nécropoles, l'architecture phénicienne combinant fréquemment les parties construites et creusées dans le roc; les sites choisis étaient situés à proximité de terroirs agricoles fertiles et étaient bien alimentés par des sources ou par une nappe phréatique peu profonde permettant de creuser des puits (telle est l'origine du nom de Beyrouth, «les puits») ${ }^{6}$.

Les cités phéniciennes paraissent avoir comporté trois parties: la zone urbaine, le territoire agricole et l'arrière-pays montagneux ${ }^{7}$. Le territoire de Byblos par exemple comprenait l'agglomération urbaine principale établie à côté du port, la plaine littorale étroite mais fertile, et la haute chaîne montagneuse portant l'ensemble cultuel d'Afqa. La documentation concernant Sidon semble indiquer que son territoire était un territoire civique, en ce sens qu'il y avait identité entre le territoire politique et les biens fonciers des citoyens; les inscriptions sidoniennes font référence à plusieurs expressions telles que "Sidon terre de la mer», «Sidon-mer», "Sidon campagne», qui ne représentaient sans doute pas de simples toponymes sui-

5 A. Poidebard, Tyr, un grand port disparu, Paris 1939; A. Poldebard et J. Lauffray, Sidon, Beirut 1951; H. Frost, "The offshore island harbour at Sidon and other Phoenician sites in the light of new dating evidence", IJNA 2, 1973, págs. 75-93; A. RABAN, "Phoenician Harbours in the Levant", Michmanim 11, 1997, págs. 7-27.

J. ElaYI et H. SAYEGH, Un quartier portuaire de Beyrouth au Fer III/Perse (en préparation).

J. ElaYI, Recherches sur les cités phéniciennes à l'époque perse, Naples 1987. 
vant le découpage naturel du territoire civique, mais désignaient plutôt des subdivisions administratives, préservant l'unité de la ville et de la campagne pour éviter de privilégier l'agglomération urbaine principale et de favoriser la prépondérance de certains éléments sociologiques ${ }^{8}$.

La zone urbaine des cités phéniciennes comprenait en général une agglomération principale donnant son nom à la cité dont elle était le centre politique, et des agglomérations secondaires, telles que Tell Qarnum/ Carné, Tartous/Antarados, Tell Ghamqé/Enhydra, Amrit/Marathos ${ }^{9}$ et Tell Kazel/Simyra pour la cité d'Arwad. Toutefois, la cité de Sidon ne semble pas avoir comporté d'agglomération urbaine principale, mais plusieurs secteurs plus ou moins urbanisés. Le territoire agricole des cités phéniciennes devait être destiné, au moins à l'origine, à subvenir à leurs besoins. Déjà à l'époque néo-assyrienne, une inscription d'Assarhaddon décrivant la cité de Sidon énumérait les différentes localités et les pâturages et terrains arrosés de son territoire agricole ${ }^{10}$. Les ressources de ce territoire ne suffisaient plus à l'époque perse pour couvrir les besoins de certaines cités comme Sidon qui furent obligées de chercher des solutions à ce problème, notamment dans l'expansion territoriale. Les territoires des cités phéniciennes pouvaient être discontinus: ainsi, celui de Tyr comprenait les villes de Crocodeilon et d'Ascalon qui étaient séparées de la ville de Tyr par les villes de Dor et de Jaffa appartenant aux Sidoniens. Ce morcellement était sans importance pour les Phéniciens, habitués au morcellement naturel, qui pouvait entraver ou même interrompre les communications terrestres, comme au Ras en-Naqura ou au Ras Cheqqa/Theouprosopon. Comme dans beaucoup de cités côtières, les communications par voie maritime étaient d'ailleurs plus aisées que par voie terrestre: ainsi, lorsque les Sidoniens et les Tyriens transportèrent des grumes de cèdres du Mont Liban jusqu'à Jérusalem ${ }^{11}$, ils n'utilisèrent pas la route terrestre directe, mais les acheminèrent d'abord jusqu'à Jaffa par mer, pour réduire au maximum la longueur du transport terrestre. Les cités phéniciennes s'étendaient aussi dans l'arrière-pays montagneux, mais on ignore jusqu'où allait leur territoire, qui ne s'étendait sans doute pas au-delà de la chaîne côtière, sauf exception, pour la cité d'Arwad par exemple. Quinte-Curce écrivait à son pro-

J. Elayl, Sidon, cité autonome de l'Empire perse, Paris 1989 (2e éd.: 1990), págs. 81-104.

Sur la cité d'Arwad, cf. J. ElAYI et M. R. HAYKAL, Nouvelles découvertes sur les usages funéraires des Phéniciens d'Arwad, Paris 1996, págs. 7-13; J. ELAYI et A. G. ELAYI, Trésors de monnaies phéniciennes et circulation monétaire (ve-IV siècles avant J.-C.), Paris 1993, págs. 24-82, et Recherches sur les poids phéniciens, Paris 1997, págs. 265-285.

10 R. Borger, Die Inschriften Asarhaddon Königs von Assyrien, AfO Beih. 9, Graz 1956, pág. 49, Nin AllI, II. 1-8.

$11+$ Rois V, 22-23; 2 Chroniques II, 15. 
pos: «En ce temps-là (c'est-à-dire à l'époque perse), le littoral et, en profondeur, une assez grande partie du pays, étaient en possession du roi de cette île, Straton" ${ }^{12}$; en effet, les villes de Mariammé, Marsya et Raphanée semblent avoir appartenu au territoire aradien à cette époque ${ }^{13}$.

L'extension du territoire des cités phéniciennes vers l'arrière-pays montagneux paraît avoir répondu à trois objectifs principaux: établir des installations cultuelles secondaires sur les montagnes voisines, assurer leur défense et contrôler les principales routes vers l'intérieur. Deir el-Qalaa, Afqa, Sfiré et Baetocécé pourraient avoir été respectivement les hauts lieux de culte de Berytos, Byblos, Tripolis et Arwad. On connaît mal l'organisation de la défense des cités phéniciennes, mais si Qalaat Sahyoun correspond bien à Sigôn, cette place-forte représentait pour les Aradiens une position stratégique importante commandant la route de la HauteSyrie par le col de Bdama et Jisr al-Sughur; de même, Mariammé contrôlait la vallée de l'Oronte de Homs à Hama ${ }^{14}$. En Phénicie centrale, quatre routes principales traversaient la chaîne montagneuse: la première suivait la vallée du Nahr ed-Damour, puis celle du Nahr Barouk et rejoignait le col de Ain Sofar, où passe actuellement la route Beyrouth-Damas. La deuxième route contournait le Djebel Niha par le sud en passant par Djezzin et Meshgara et rejoignait la vallée du Litani; la troisième suivait le Nahr Zaharani et, par Nabatiyé et Arnoun, rejoignait également la vallée du Litani; la quatrième suivait la vallée du Litani. Mais les communications avec le couloir Oronte-Beqaa-Jourdain se faisaient plus facilement au nord et au sud de la Phénicie centrale; le sud de la Phénicie du sud était enfin le point de départ de la route vers le golfe d'Aqabah et l'Arabie, le long du Wadi Ghazzeh. La faible superficie des territoires des cités phéniciennes est comparable à celle des cités grecques: le territoire de Tyr (sans compter ses possessions en Phénicie du sud) était sans doute un peu plus petit que celui d'Athènes, que l'on estime à environ 2500 $\mathrm{km}^{2}$; il n'y a en effet que $75 \mathrm{~km}$ entre le Litani et le Kishon, deux cours d'eau qui pourraient avoir constitué les limites naturelles nord et sud, et l'on peut penser que la largeur du territoire de Tyr représentait moins de la moitié de sa longueur.

La royauté constituait un élément caractéristique de ces cités-Etats. Le terme $M L K$ désignait à la fois le roi et son équivalent divin. La royauté

\footnotetext{
12 Quinte-Cunce, Histoires IV, 1, 6.

13 J.-P. Rey-CoQuals, Arados et sa pérée, Paris 1975.

14 R. Dussaud, Topographie historique de la Syrie antique et médiévale. Paris 1927; ELAYI, Economie..., págs. 9-57.
} 
phénicienne était unique, viagère et héréditaire; cependant, la mère du roi pouvait exercer une co-régence si son fils était encore trop jeune pour gouverner seul, ou bien le roi régnant pouvait, sur la fin de son règne, associer le prince héritier à ses fonctions politiques. Ainsi, le roi de Sidon Bodashtart était soucieux de légitimer son fils héritier Yatonmilk en l'associant à la fonction de bâtisseur, sans doute à cause des difficuliés successives survenues dans la dynastie d'Eshmunazor 15: mort prématurée de Tabnit, exercice du gouvernement par la mère du jeune roi et mort prématurée d'Eshmunazor II qui était apparemment sans héritier car Bodashtart était issu d'une branche collatérale. De même, le roi d'Arwad Gérostratos pourrait avoir associé son fils Straton, prince héritier, à certaines de ses fonctions royales. La co-régence du roi régnant avec le prince héritier est un phénomène connu chez les autres Sémites occidentaux, l'exemple le plus célèbre étant celui de Salomon, proclamé roi sous le règne de son père David. On a aussi l'exemple d'Athalie, fille d'Achab, qui régna pendant près de sept ans sur Juda après l'assassinat de son fils Akhazias. La mère du roi de Sidon Eshmunazor II participait aux fonctions politiques telles que la construction de temples, la réception des villes de Dor et de Jaffa et leur intégration dans le territoire de Sidon, participation reconnue non seulement par son fils mais par le roi des Perses; Eshmunazor portait le titre de "roi des Sidoniens" tandis que sa mère était appelée simplement "la reine", mais elle avait seule le privilège d'être "prêtresse d'Ashtart". Toutefois, le rôle exceptionnel qu'Amoashtart joua dans la royauté sidonienne s'explique sans doute par des circonstances particulières: en effet, Eshmunazor se dit "orphelin, fils d'une veuve", ce qui signifie sans doute qu'il était né après la mort de son père Tabnit; c'est donc sa mère, dont l'importance était renforcée par le fait qu'elle était à la fois la femme et la soeur (ou la demi-soeur) de Tabnit, qui exerça la co-régence pendant la minorité de son fils. Le phénomène de la co-régence n'est pas attesté à Byblos, mais l'inscription funéraire de Batnoam, mère du roi Ozbaal, usurpateur fils du prêtre Paltibaal, fait apparaître l'importance de son rang à travers la solennité et la richesse de son inhumation; bien qu'elle ne fût pas de sang royal, elle fut traitée comme les autres mères de rois de sang royal, peut-être en récompense du rôle qu'elle avait pu jouer dans l'usurpation de son fils. L'usage sémitique d'indiquer la titulature royale dans les inscriptions permet de savoir que la royauté phénicienne était héréditaire et, de ce fait, probablement viagère comme l'indiquent les sources classiques: "Nul n'était admis à ce haut rang s'il n'était pas issu de souche

15 Sur la datation de la dynastie d’Eshmunazor, cf. ELAYI, Sidon..., págs. 235-243; id., Trans 11, 1996, págs. 180. 
royale" ${ }^{16}$. La succession par le fils est indiquée dans la titulature de plusieurs rois de Sidon et de Byblos jusqu'à la deuxième ou troisième génération. Elle ne se faisait pas nécessairement par ordre de primogéniture: ainsi, Bodashtart de Sidon était le fils du frère (ou demi-frère), ou de la soeur (ou demi-soeur) d'Amoashtart et de Tabnit. Lorsqu'Alexandre choisit pour remplacer le roi de Sidon, non pas son fils, mais un autre membre de la famille royale sidonienne, il respectait sans doute la procédure locale comme il le faisait d'ordinaire dans ses conquêtes. On a plusieurs renseignements sur l'existence de ruptures dans les dynasties phéniciennes, en particulier à Byblos: Yehawmilk et Ozbaal n'attribuent pas à leur père un titre royal dans leur généalogie, ce qui indique une usurpation probable.

Les fonctions politiques des rois phéniciens dont on a quelque connaissance pour l'époque perse sont les fonctions sacerdotale, guerrière et peut-être judiciaire. La fonction sacerdotale est la mieux attestée et il est probable qu'elle a été la plus importante, surtout dans certaines cités. Ainsi, la royauté était à Byblos de droit divin, en ce sens que le roi affirmait devoir le trône à une divinité qu'on pourrait appeler dynastique: «Je suis Yehawmilk, roi de Byblos, que la Dame, patronne de Byblos, a fait roi sur Byblos" 17. La place accordée au titre sacerdotal dans la titulature des rois de Sidon comme Tabnit et Eshmunazor ler semble indiquer qu'il recouvrait une fonction religieuse prioritaire. Même lorsque le roi ne portait pas de titre sacerdotal, il prenait en charge les travaux de construction, réfection ou décoration des édifices religieux de la cité; l'activité de bâtisseur est bien attestée par exemple pour Bodashtart de Sidon, qui enterrait rituellement dans les fondations des inscriptions commémoratives en plusieurs exemplaires. II pouvait aussi instaurer de nouveaux lieux de culte, régler certaines pratiques religieuses et accomplir personnellement des actes sacerdotaux, mais on ne saurait préciser l'étendue de son rôle dans le culte. La fonction guerrière du roi phénicien n'est pas exprimée par un titre particulier et n'est même pas mentionnée dans les inscriptions phéniciennes, mais on la connaît indirectement par les sources classiques et les représentations des monnaies. II commandait la flotte de guerre de sa cité, non pas de manière théorique mais il devenait en temps de guerre un véritable amiral qui préparait la stratégie navale, par exemple dans le conseil de guerre de Xerxès avant Salamine, et participait aux combats. Cette fonction guerrière semble avoir été assez importante et c'est à tort qu'on se représente toujours les Phéniciens comme des commerçants et des marins

6 QUINTE-CuRCE, Histoires IV, 1, 17-18.

17 H. DONNER et W. ROLLIG, Kanaanäische und aramäische Inschriften, Wiesbaden 1962-64, $n^{\circ} 10$ 
réputés, jamais comme de bons soldats. Pendant les guerres médiques, de l'aveu même des auteurs grecs, les Phéniciens ont apporté la preuve que leurs flottes pouvaient rivaliser avec celle d'Athènes. Leur habileté dans le combat terrestre ne le cédait en rien à leur habileté dans la stratégie navale comme en témoigne la défaite infligée par les Sidoniens aux armées satrapiques de Bélésys et de Mazaios/Mazday. Ils excellaient aussi dans les travaux militaires et le système de défense des cités phéniciennes était très perfectionné; ils étaient aussi bien pourvus de machines défensives qu'offensives les plus sophistiquées, qui sont décrites par Arrien dans le récit du siège de Tyr par Alexandre ${ }^{18}$. Une telle maîtrise de l'art militaire ne saurait s'expliquer seulement par l'encouragement des rois perses pour qui les flottes phéniciennes constituaient la base de leur puissance militaire en Méditerranée: elle supposait une longue expérience militaire, illustrée par exemple dans les symboles guerriers des monnaies phéniciennes. Quelques indices montrent que le roi phénicien avait aussi une fonction judiciaire, qui reste à préciser car les suffètes attestés à Tyr à cette époque n'exerçaient pas de pouvoir politique.

Bien que le roi ait assumé les fonctions sacerdotale, guerrière et peutêtre judiciaire, la royauté phénicienne n'avait rien d'une tyrannie car les pouvoirs du roi semblent toujours plus ou moins limités. Certains indices montrent qu'il y avait, à côté du pouvoir royal, un ou plusieurs organismes représentatifs de la communauté civique, mais leur identification n'est pas facile car les sources sont hétérogènes et lacunaires. La diversité et la confusion qui caractérisent la terminologie, aussi bien dans les sources classiques que dans l'Ancien Testament, et les informations que nous avons sur la période précédente, permettent de penser que les structures du pouvoir politique étaient originales et complexes, et laissent apparaître au moins deux niveaux de représentation. Le roi n'avait pas le monopole des fonctions qu'il exerçait, mais il les partageait toutes, à des degrés divers, avec les citoyens de sa cité et la séparation des pouvoirs ne paraît pas avoir eu de sens dans la pensée phénicienne; la faible superficie des cités phéniciennes devait favoriser la participation des citoyens aux affaires publiques. A côté du pouvoir royal, certains membres de la famille tels que le fils prince héritier et la mère du roi pouvaient aussi détenir un pouvoir politique qui s'exerçait par exemple sous la forme d'une association à certains actes politiques dans le cas du fils, et sous la forme d'une co-régence dans le cas de la mère. Le corps civique comportait plusieurs niveaux de représentation, qui s'exprimaient dans des organismes hiérarchisés,

${ }^{18}$ ARRIEN, Anabase d'Alexandre II, 15-16. 
encore mal connus: il y avait peut-être un "conseil des Anciens", bien attesté pour la période précédente, et un "conseil des Cent", présidé par un "chef des Cent", dont les principaux membres du clergé, notamment le "chef des prêtres", et les "suffètes", faisaient vraisemblablement partie; l'inscription de Yehawmilk de Byblos paraît se référer à une «assemblée du peuple" dont le roi devait conserver la faveur. L'équilibre entre le pouvoir royal et les organismes représentatifs, ou entre ces différents organismes, était souvent précaire. Dans quelques cas apparemment exceptionnels, un conseil restreint a pu intervenir dans le choix d'un nouveau roi, et la décision a pu être ensuite simplement entérinée par le peuple ou l'assemblée du peuple.

Seuls les citoyens semblent avoir eu une part active et légale à la vie de l'Etat en participant aux institutions politiques, militaires, judiciaires et religieuses; ils ne représentaient pas la société de la cité-Etat dans son acception sociologique, mais ne constituaient qu'une partie, probablement minoritaire, de l'humanité vivant sur le territoire civique; en dehors de la reine-mère, on ignore si les femmes avaient accès à la citoyenneté. Les droits civiques semblent avoir connu dans les cités phéniciennes des extensions variables selon les cités et les époques, ces différenciations étant à la base de la différenciation des régimes politiques. Le droit de cité était non seulement restrictif mais hiérarchisé, une partie des citoyens seulement jouissant de la totalité des droits civiques. L'accès à chacun des organismes représentatifs pourrait avoir été limité par un numerus clausus ("conseil des Cent"?). Parmi les critères d'accès à la citoyenneté, qui pouvaient se combiner de différentes manières, le plus évident était celui de la naissance car la tendance aristocratique persistait à l'époque perse, plus ou moins marquée selon les cités: le roi et certains membres de la famille royale constituaient le sommet de la hiérarchie sociale; les "Grands" devaient être des citoyens de plein droit appartenant aussi à un organisme représentatif restreint. Le critère de l'âge était peut-être pris en considération dans la mesure où il y avait encore un "conseil des Anciens". Le critère du rang social pourrait aussi avoir été retenu comme semblerait l'indiquer la puissance du haut clergé et des suffètes, de même que le critère de la fortune dans la mesure où les riches citoyens étaient astreints à financer les préparatifs de guerre.

Les systèmes politiques phéniciens étaient des systèmes complexes, vraisemblablement mixtes, comportant de nombreuses variantes, et irréductibles à aucun autre, si proche fût-il. La royauté, qui constituait l'élément caractéristique, s'apparentait dans une certaine mesure aux royautés voisines: ainsi, certains aspects de l'idéologie royale phénicienne sur les travaux de construction et les rites de fondation, le sens de l'apparat et le 
goût de la chasse rappellent l'idéologie royale assyro-babylonienne. Les problèmes de succession et les ruptures dynastiques se retrouvent dans la plupart des royautés de la région. Le système de la co-régence et la place accordée au prince héritier font surtout penser aux royaumes d'Israël et de Juda. La limitation du pouvoir royal par des organismes représentatifs se retrouve au premier millénaire dans toutes les royautés de la région qui n'étaient pas des monarchies militaires centralisées, par exemple à Kanish, à Babylone, Sippar ou Nippur. Malgré des traits communs indéniables, les cités-Etats phéniciennes se différenciaient aussi bien de la citéEtat d'Ugarit et des cités-temples néo-babyloniennes que du royaume d'Israël et de la province de Judée, et se rapprochaient des poleis, citésEtats grecques, par le fait que leur territoire était un territoire civique, en ce sens qu'il y avait identité entre le territoire politique et les biens fonciers des citoyens, et que tous les citoyens participaient plus au moins au pouvoir politique à travers les organismes représentatifs. La hiérarchisation du corps civique, dont les critères n'ont pas été seulement celui de la naissance, semble révéler une tendance oligarchique. II pourrait y avoir eu, dans quelques cas et à un moment donné, une assemblée du peuple assez élargie participant au pouvoir politique, ceci restant encore indémontrable dans l'état actuel de la documentation. Au total, les institutions politiques des cités phéniciennes s'insèrent bien dans le cadre des autres institutions nord-ouest sémitiques, mais pour diverses raisons, ces cités ont été amenées à développer un type particulier de cités-Etats dont on ne connaît pas pour le moment de parallèles dans la région, et qui s'apparentent par certains côtés aux cités-Etats grecques, sans que l'on puisse, dans l'état actuel de la documentation, déceler d'emprunts ni dans un sens ni dans l'autre. II s'agit d'un développement politique original dont on ignore les causes, et que ni les orientalistes ni les hellénistes n'ont encore mis en évidence.

Ce schéma des institutions politiques phéniciennes que j'ai proposé serait incomplet si l'on ne tenait pas compte des variantes locales, des évolutions au cours de cette période et de leur insertion dans le cadre de la domination perse. Parmi les différences politiques entre les cités, on notera par exemple des différences de titulatures qui impliquent des différences dans les fonctions royales, et une certaine diversité dans les organismes représentatifs qui suppose un équilibre variable entre les pouvoirs. La politique extérieure des cités a connu des divergences dont on peut juger en comparant leurs réactions à l'arrivée d'Alexandre, car elles ne subissaient plus à ce moment-là la pression politique perse. Une différence importante entre les cités phéniciennes a été aussi la différence de richesse qui paraît correspondre au moins en partie à une différence de puissance et 
d'autonomie; seules les cités les plus puissantes, Sidon, Tyr et Arwad, ont pu s'étendre et exercer leur contrôle en Phénicie du nord et en Phénicie du sud; à l'époque perse, Sidon a exercé son hégémonie sur les autres cités, relayée vraisemblablement par Tyr vers la fin de cette période. Tripolis paraît avoir été une cité phénicienne originale, d'après les sources grecques qu'il faut utiliser avec prudence en tant que sources extérieures ${ }^{19}$. Son originalité provenait apparemment du fait qu'elle était formée de trois villes dépendant politiquement des trois grandes cités phéniciennes, Sidon, Tyr et Arwad; le fait que chacune possédait son enceinte fortifiée semble impliquer qu'elle avait aussi son propre gouvernement, plus ou moins contrôlé par la cité à laquelle elle appartenait; elle aurait été le siège d'un conseil fédéral qui y aurait tenu épisodiquement des assemblées générales, sans permanence ni régularité, où toutes les cités alliées auraient été représentées dans une "assemblée générale des Phéniciens". On signalera la prépondérance de Sidon dans la confédération de Tripolis et l'existence de symmachies, alliances militaires peut-être assez structurées. II est possible enfin que la cité-Etat ne soit pas la seule forme de pouvoir politique phénicien dans cette région, notamment en Phénicie du sud et en Phénicie du nord où bien des zones d'ombre subsistent encore.

L'évolution des cadres politiques des cités phéniciennes est encore plus difficile à saisir que les différences locales. Le développement de la citéEtat, dont la naissance est antérieure à la période perse, n'était pas aussi avancé dans toutes les cités qui étaient alors en plein devenir. Ainsi, sous le dernier roi de Byblos, Aynel, l'évolution des légendes monétaires, de la titulature royale complète au nom de la cité, pourrait indiquer un affaiblissement du pouvoir royal au profit des organismes représentatifs ${ }^{20}$. Après la répression de la révolte de Sidon par Artaxerxès III vers 350 , la disparition d'un grand nombre de Sidoniens, en particulier de membres des organismes représentatifs, et la pression politique perse consécutive à la révolte pourraient avoir conduit à l'instauration d'un régime tyrannique sous Straton II.

On se gardera d'oublier en effet que les pouvoirs locaux et l'organisation du territoire des cités phéniciennes s'inséraient dans le cadre de l'Empire perse achéménide ${ }^{21}$. En fait, l'histoire des cités phéniciennes au premier millénaire avant notre ère s'inscrit dans le cadre permanent de la

19 J. ELAYI, “Tripoli (Liban) à l'époque perse», Trans 2, 1990, págs. 59-71

20 Cf. ELAYI-ELAYI, Trésors..., págs. 83-124.

21 J. ELAY1, "Les cités phéniciennes entre liberté et sujétion", DHA 16, 1990, págs. 93-113; id., Sidon..., págs. 137-159; P. Briant, Histoire de l'Empire perse de Cyrus à Alexandre, Paris 1996. 
domination des Empires qui se sont succédé au Proche-Orient: assyrien, néo-babylonien, perse, grec et romain. Depuis la véritable «rupture historique» que constitue le règne du roi assyrien Tiglath-phalazar III (744-727), elles étaient passées d'une période d'indépendance à un statut d'autonomie relative pour certaines d'entre elles et d'assujettissement total pour d'autres; les bases du système impérialo-tributaire qui fut repris par les Perses étaient jetées: justification idéologique du système, création d'un Etat fortement structuré à ambitions continentales et maritimes, politique permanente de conquête, généralisation du système tributaire, mise en place d'une couverture administrative et militaire dans tout l'Empire, développement des communications. Certes, il restait à vaincre les résistances locales, à consolider partout et à optimiser le système: la dynastie néo-assyrienne des Sargonides joua un rôle fondamental à cet égard, l'Empire néo-babylonien poursuivit le développement du système, de même que l'Empire perse qui l'optimisa. Ainsi, le contrôle territorial représentait un problème essentiel pour un Empire construit par la conquête militaire et sans cesse menacé par des sécessions: les Perses développèrent et améliorèrent le réseau routier, en particulier sur le plan de la sécurité (garnisons et postes de garde) et des capacités logistiques pour le transfert des troupes d'une région à l'autre (stocks de vivres, dépôts d'eau, réserves d'armes). Le contrôle territorial s'exprimait, non seulement par un système efficace de communications, mais aussi par un réseau de places-fortes et de garnisons: les Perses firent un effort particulier dans ce domaine pour développer la défense du front méditerranéen de l'Empire, devenue plus indispensable que sous leurs prédécesseurs, du fait de l'expansion accrue vers l'ouest.

L'amélioration du contrôle territorial permettait un meilleur contrôle politique et une plus grande exploitation des richesses. Le versement du tribut devint effectivement annuel à la fin du règne de Darius, phénomène nouveau qui dut affecter la vie économique des cités phéniciennes. En dehors du tribut, elles étaient soumises à d'autres taxes, déjà en vigueur mais dont certaines furent alourdies. L'exploitation des ressources forestières s'était accrue du fait de l'extension des domaines royaux, essentiellement forestiers, du développement du réseau routier qui les rendaient plus accessibles, et de l'augmentation des besoins. En effet, si la fourniture de bois n'avait guère changé dans la construction et la restauration des palais par rapport aux Empires précédents, elle augmenta de manière considérable dans les arsenaux maritimes. Le développement de la guerre sur mer sous l'Empire perse obligea les Phéniciens à construire de puissantes flottes de guerre au service de l'occupant et à les reconstituer après les défaites, qui furent fréquentes au cours de la première moitié du ve siè- 
cle. Les cités phéniciennes ne constituaient plus la limite occidentale de l'Empire comme avant le règne de Sargon II, mais des points d'accès au monde occidental en partie conquis et en partie à conquérir. Plusieurs d'entre elles représentaient par conséquent pour les Perses des positions stratégiques pour contrôler la route côtière et la mer, ou des bases d'opérations vers l'Egypte et les cités grecques, avec la lourde charge d'entretenir des résidents perses et des troupes de garnison, augmentées ponctuellement de troupes auxiliaires. Elles possédaient des atouts essentiels qui imposaient à leurs conquérants de les ménager: les flottes phéniciennes représentaient la base de la puissance navale nécessaire au maintien de l'Empire, les sites côtiers phéniciens étaient des positions stratégiques, et leur prospérité économique constituait une abondante source de profit, indispensable au bon fonctionnement du système impérialo-tributaire. L'habileté des conquérants assyro-babyloniens et perses consista, non pas à s'approprier ces atouts, mais à laisser les cités phéniciennes autonomes pour les gérer au mieux de leurs intérêts, sachant qu'ils récolteraient de cette manière de meilleurs bénéfices: ainsi, les flottes de guerre de ces cités étaient construites par leurs artisans dans leurs arsenaux, montées par des équipages locaux et commandées par leurs rois; chaque cité avait son armée et son système de fortifications, elle exploitait ses ressources agricoles et organisait à sa guise ses activités commerciales. Les autres aspects de la vie des cités, institutionnel, social, religieux et culturel par exemple, intéressaient peu les occupants; il leur suffisait, en temps normal, de surveiller les pouvoirs locaux, afin qu'ils ne portassent pas atteinte aux intérêts de l'Empire. Mais cette ligne politique dut être corrigée à diverses reprises à cause des incidents de parcours comme les révoltes, avec des conséquences plus ou moins lourdes pour les cités phéniciennes.

Un passage de l'inscription d'Eshmunazor, roi de Sidon, illustrera pour finir la complexité des pouvoirs et de leurs relations, ainsi que l'insertion de l'économique dans le politique: "Le seigneur des rois nous a donné Dor et Jaffa, les puissantes terres à blé qui sont dans la plaine du Sharon, en récompense des actes remarquables que j'ai accomplis, et nous les avons ajoutées aux frontières du territoire afin qu'elles appartiennent aux Sidoniens pour toujours" ${ }^{22}$. Ce don ne constituait pas un simple transfert de droits politiques, mais entraînait une nouvelle source de revenus car l'inscription souligne la valeur agricole de ces terres; inversement, des terres à blé sont définies en termes de puissance politique. Ce type d'attribution de terres, qui devint fréquent à l'époque hellénistique, montre que le roi

22 Donner-RÖllig, Inschriften..., n 14; cf. Elayi, Economie..., págs, 60, 72. 
des Perses avait le droit de disposer des terres de l'Empire, sans contradiction apparente avec l'existence de la propriété civique, communautaire ou privée. Il faisait ce don personnel au roi Eshmunazor et à sa mère Amoashtart dans la pensée qu'ils allaient les ajouter au domaine du palais, mais le roi de Sidon et la reine-mère transformèrent ce don personnel en don aux Sidoniens car il n'y avait plus d'économie palatiale distincte de celle de la cité; leur position était sans doute incontortable car ils étaient obligés de mener un jeu subtil entre leurs deux identités politiques: pouvoir royal fort aux yeux des Perses avec économie palatiale séparée, et pouvoir politique limité aux yeux de leurs concitoyens. 


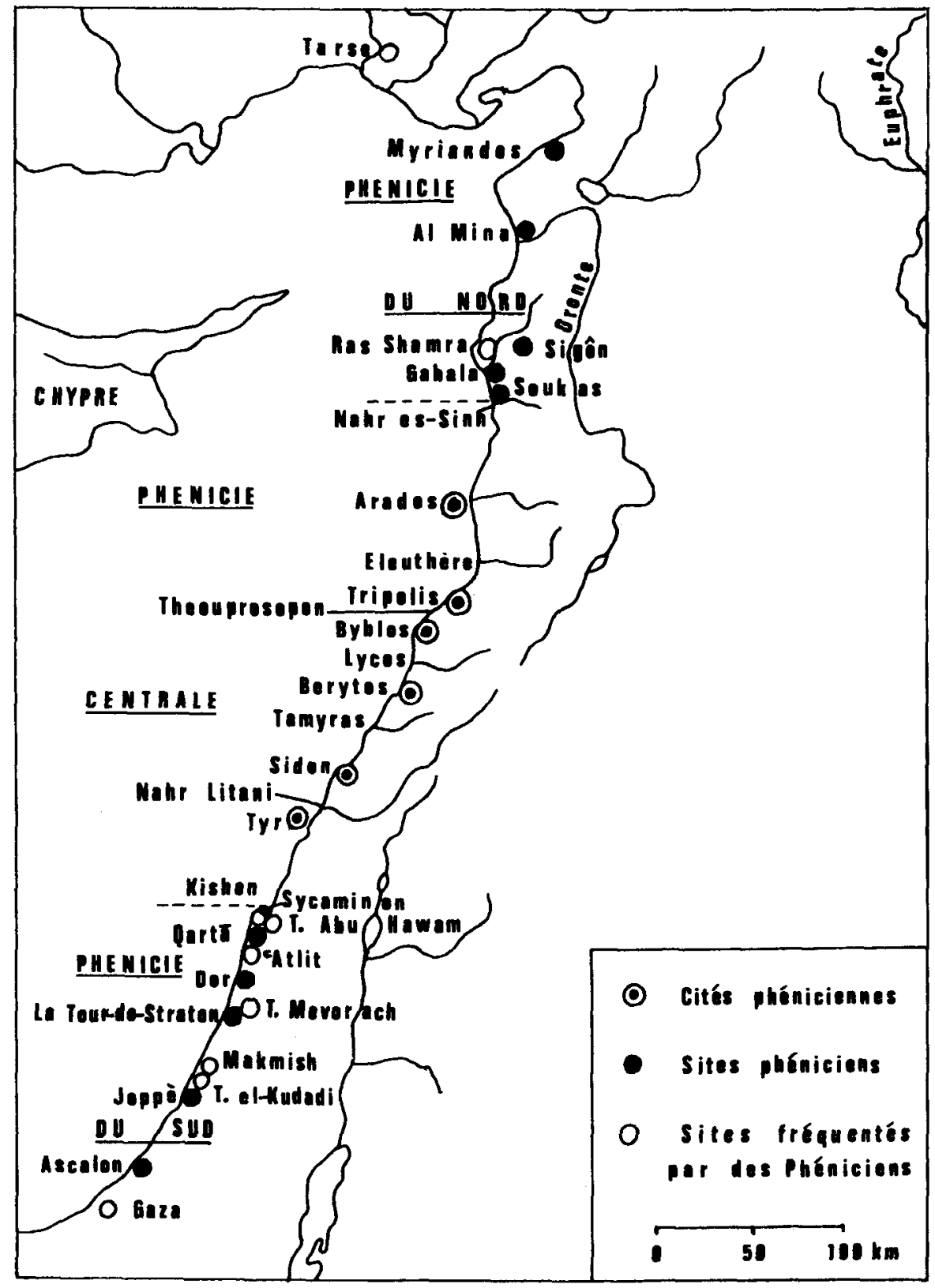

Les Phéniciens en Méditerranée orientale à l'Époque perse. 
